\title{
OSTEOARTHRITIS
}

\section{Bow-legged or knock-kneed, MRI studies probe cartilage damage in relation to knee alignment and the risk of $O A$}

T

wo epidemiological studies using

large US datasets, published in Annals

of the Rheumatic Diseases, offer

insights into the relationships between

knee joint alignment, cartilage wear and osteoarthritis (OA). Both studies used MRI to compare patterns of cartilage lesions between people with normal, varus (bowlegged) or valgus (knock-kneed) alignment. Leena Sharma and colleagues looked for incident tibiofemoral cartilage damage in previously healthy knees, whereas Doug Gross and co-workers examined patellofemoral damage patterns in relation to knee alignment. The studies might have implications for noninvasive management of knee OA.

"It is fairly well established," explains Sharma, "that, in persons with established knee OA, varus alignment is associated with subsequent progression of medial tibiofemoral $\mathrm{OA}$ and valgus with progression of lateral OA.” Results from previous radiographic studies have suggested that malalignment might increase the incidence of knee OA, but have not been conclusive.

To gain a clearer picture of whether knee alignment is a risk factor for the development of OA, Sharma and colleagues examined MRI images of knees from people enrolled in the Multicentre Osteoarthritis (MOST) study. This prospective cohort includes patients with $\mathrm{OA}$ and people considered to be at high risk of the disease. Knees without damage at baseline were selected (293 joints from 256 people), and damage at 30 months was assessed by MRI. Alignment was assessed by weightbearing radiography of the lower extremity, and associated with cartilage morphology at baseline and follow-up.

"Varus (as compared with non-varus) was associated with incident medial damage, as was varus versus neutral, with evidence of a dose effect (that is, greater risk with more severe varus)," reports Sharma. Varus alignment was associated with a reduced risk of incident lateral damage.

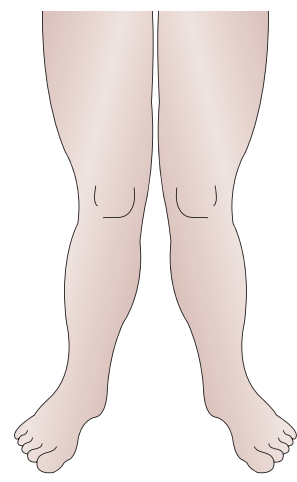

Genu valgum

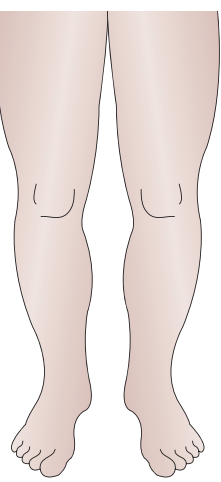

Normal

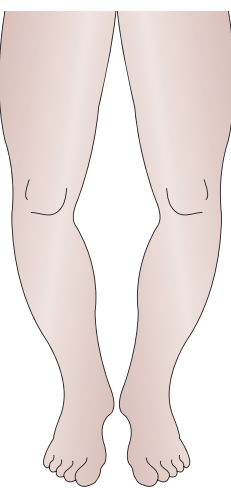

Genu varum

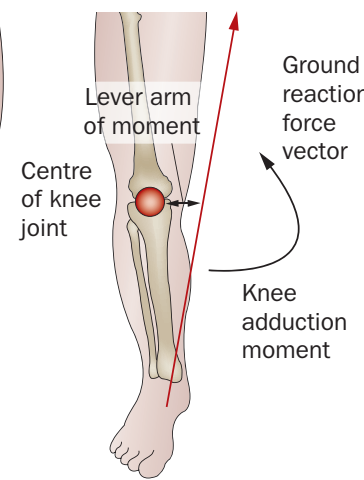

From Roos, E. M. et al. Nat. Rev. Rheumatol. 7, 57-63 (2011).

Valgus alignment was associated with reduced incident medial damage, but was not associated with incident lateral damage. These findings, adds Sharma, "uniquely support that varus alignment is associated with a greater risk of incident knee OA." They also support the investigation and application of noninvasive preventive strategies such as orthoses and gait modification. "In an individual with a varus knee, it is possible that an intervention which improves medial to lateral load distribution could help to delay the onset of OA; at this early point, such a strategy may be more powerful than when OA has established its presence in a knee," says Sharma.

Ewa Roos, an expert in the role of muscle function and exercise in prevention and treatment of OA (who was not involved in the study) agrees that interest in noninvasive strategies will increase. "Non-surgical options include neuromuscular training that aims at improving sensorimotor control and achieving compensatory functional stability," she adds, explaining that the training aims to maintain good alignment, and has resulted in improved cartilage quality in patients at high risk of OA.

Of less clear clinical significance but intriguing to $\mathrm{OA}$ epidemiologists are the findings of Gross and colleagues, whose "simple" analysis used MRI images from the MOST study, as well as the Boston OA of the knee and Framingham OA studies, to examine patellofemoral OA (PFOA). Their results challenge a presumption-which follows from the 'Law of Valgus' (dictating the direction of force distribution on the patella) - that medial PFOA arises only rarely, and then only as a consequence of varus deformity. "Medial PF cartilage damage is undeniably prevalent in three of the largest OA studies available," says Gross, "and its prevalence remains high even among knees that do not have varus deformity". He suggests the finding might explain anecdotal evidence that medial patellofemoral pain is reported by patients more frequently than expected.

David Hungerford, who jointly introduced the 'Law of Valgus', notes, however, that progressive OA is distinct from cartilage abnormalities. "In patients with progressive symptomatic PFOA," he says, "the law of valgus remains intact".

\section{Emma Leah}

Original articles Sharma, L. et al. The role of varus and valgus alignment in the initial development of knee cartilage damage by MRI: the MOST study. Ann. Rheum. Dis. doi:10.1136/annrheumdis-2011-201070 | Gross, K. D. et al. Breaking the Law of Valgus: the surprising and unexplained prevalence of medial patellofemoral cartilage damage. Ann. Rheum. Dis. doi:10.1136/ annrheumdis-2011-200606 Further reading Roos, E. M. et al. Muscle weakness, afferent sensory dysfunction and exercise in knee osteoarthritis. Nat. Rev. Rheumatol. 7, 57-63 (2011) 